\title{
Yiqihuoxuejiedu Formula Restrains Vascular Remodeling by Reducing the Inflammation Reaction and Cx43 Expression in the Adventitia after Balloon Injury
}

\author{
Hong Chang, ${ }^{1}$ Huan Lei, ${ }^{1}$ Yizhou Zhao, ${ }^{1}$ Ruixue Yang, ${ }^{1}$ Aiming Wu, ${ }^{1}$ Yingqiu Mao, \\ Youliang Huang, ${ }^{2}$ Xiying Lv, ${ }^{1}$ Jiuli Zhao, ${ }^{1}$ Lixia Lou, ${ }^{1}$ Dongmei Zhang, ${ }^{1}$ Yingkun He, \\ Ying Xu, ${ }^{1}$ Tao Yang, ${ }^{1}$ and Mingjing Zhao ${ }^{1}$ \\ ${ }^{1}$ Key Laboratory of Chinese Internal Medicine of Ministry of Education and Dongzhimen Hospital, \\ Beijing University of Chinese Medicine, Beijing 100700, China \\ ${ }^{2}$ Beijing University of Chinese Medicine, Bei San Huan Dong Lu 11, Chao Yang District, Beijing 100029, China
}

Correspondence should be addressed to Mingjing Zhao; mjgx2004@163.com

Received 6 May 2015; Revised 30 July 2015; Accepted 9 August 2015

Academic Editor: Dan $\mathrm{Hu}$

Copyright ( $) 2015$ Hong Chang et al. This is an open access article distributed under the Creative Commons Attribution License, which permits unrestricted use, distribution, and reproduction in any medium, provided the original work is properly cited.

\begin{abstract}
Vascular remodeling is closely related to hypertension, atherosclerosis, and restenosis after PCI. Considerable evidence indicates that the activation and proliferation of adventitial fibroblasts play key roles in vessel injury. The inflammatory response and high expression of connexins contribute to adventitial remodeling. Therefore, reducing inflammation reaction and connexins expression in adventitia may become a new target to prevent vascular remodeling. Yiqihuoxuejiedu formula, composed of TCM therapeutic principle of supplementing qi, activating blood and detoxification, can inhibit restenosis after intimal injury. To further investigate the effect of Yiqihuoxuejiedu formula on inflammation and connexins, we established a carotid artery injury model. In model rats, hyperplasia in the intima was mild but obvious in the adventitia; CRP heightened; expressions of MCP-1, CD68, and Cx43 increased. Yiqihuoxuejiedu formula relieved intimal hyperplasia and adventitial area, obviously diminished the expressions of CD68 and $\mathrm{Cx} 43$ in the adventitia, and reduced CRP but did not lower MCP-1. These results indicated that Yiqihuoxuejiedu formula inhibited vascular remodeling especially adventitial hyperplasia by reducing the inflammation reaction including lowering macrophages infiltration and systemic nonspecific inflammatory response and also restraining gap junction connexins leading to less communication among cells. This study provides new ideas and methods for the prevention and treatment of vascular remodeling.
\end{abstract}

\section{Introduction}

Vascular remodeling is a structural and functional variation of vessels to adapt to the intracorporal environment. For a long time, vascular smooth muscle cells (VSMCs) in the media have been regarded as a central link and the adventitia has been known to play only supportive functions [1]. However, the adventitia is an essential regulator of vascular wall structure and function. Adventitial fibroblasts (AFs, the major component of the adventitia) are activated and transfer into myofibroblasts, proliferate, and migrate to media and intima to participate in the progression of vascular remodeling $[2,3]$.
In the initial stages of intimal balloon injury, one of the key triggers of vascular remodeling is early inflammation in the adventitia [4] including the infiltration of macrophages [5] and neutrophils [6] and the release of inflammatory factors, such as interleukin- (IL-) $1 \beta$, IL-6, IL-8, and MCP-1 $[7,8]$. Research in patients also found that in-stent restenosis is related to macrophage infiltration [9]. Meanwhile myofibroblasts release various proinflammatory cytokines, for instance, MCP-1, recruiting macrophages and neutrophils to infiltrate into the adventitia $[10,11]$. These inflammatory responses promote activation and proliferation of adventitial fibroblasts, resulting in adventitial remodeling. 
Cellular interaction in blood vessels is maintained by multiple communication pathways, including gap junctions. Gap junctions (GJs) arise from the docking of two hemichannels or connexons, formed by the assembly of six connexins (Cxs), and achieve direct cellular communication by allowing the transport of small metabolites, second messengers, and ions between two adjacent cells [12]. Although Cx37, Cx40, and $\mathrm{Cx} 43$, respectively, are expressed in different layers of vessel wall, Cx43 is common in all the three layers [1316]. Accumulating evidence supports the results that $\mathrm{Cx} 43$ has been deeply investigated in cardiovascular diseases. In atherosclerosis, $\mathrm{Cx} 43$ promoted leukocyte to adhere to endothelium and infiltrate into the media, which deteriorated atherosclerosis [17]. Further investigation showed that reduced $\mathrm{Cx} 43$ expression could inhibit atherosclerotic lesion formation in low-density lipoprotein receptor-deficient mice [18]. Meanwhile, upregulation of Cx43 promoted SMC phenotypic transformation and accelerated intimal hyperplasia, leading to vascular restenosis $[19,20]$. In view of the function of $\mathrm{Cx} 43$, its expression and relationship with inflammation in the adventitia need to be illustrated.

In 2011 PCI guideline, treatment and prevention after PCI mainly include antihypertensive therapy including $\beta$ receptor blockers, angiotensin-converting enzyme inhibitors (ACEI), lipid-lowering therapy with statins, and antiplatelet/anticoagulant therapy with aspirin and clopidogrel [21]. Of these drugs, statins not only lower lipid concentration but also diminish inflammation and expression of GJ [22, 23]. Though western medicine has gained success, a number of potential risks remain, and we need to find new drugs to prevent vascular remodeling.

Myofibroblasts play pivotal role in the tissue repair and remodeling and are also a key player in pathological hypertrophic scars and organ fibrosis. The vascular remodeling after PCI is similar to local wound repair. Based on this idea, as well as the traditional Chinese medicine treatment of supplementing qi, activating blood plus detoxification, Yiqihuoxuejiedu formula was prescribed to prevent and treat vascular remodeling. The prescription is composed of astragalus, salvia, honeysuckle, and other components. Previous researches have shown that the prescription can reduce vessel stenosis, lower blood lipids [24], inhibit the activation and proliferation of the adventitial fibroblasts, and decrease collagen content and type I/III collagen ratio in the adventitia [25].

In this study, we used a vascular remodeling model of intimal injury with balloon injury and made Atorvastatin a positive control to explore the underlying mechanism of Yiqihuoxuejiedu's inhibition on adventitial inflammation and regulation of GJs at the early stages of injury (7 days). This study may provide a new approach to prevent vascular remodeling.

\section{Materials and Methods}

2.1. Animals. Male Sprague-Dawley (SD) rats weighing 380 to $420 \mathrm{~g}$ were purchased from Beijing Weitong Lihua Experimental Animal Technology Co., Ltd., Beijing, China (certificate number SCXK (Beijing) 2012-0001). Rats were raised in SPF room in Beijing University of Chinese Medicine. All the procedures were conformed to the National Institute of Health's Guide for the Use and Care of Laboratory Animals.

2.2. Establishment of Balloon Injury Model. One hour before anesthesia, a deep subcutaneous injection of heparin was given to each rat by $500 \mathrm{U} / \mathrm{kg}$. Next, rats were anesthetized with $40 \mathrm{mg} / \mathrm{kg}$ sodium pentobarbital. As showed in the past study [25], a Fogarty $2 \mathrm{~F}$ balloon catheter (diameter of balloon $2 \mathrm{~mm}$, length $20 \mathrm{~mm}$, Medtronic Company, USA) was introduced through the left external carotid artery and advanced $4-4.5 \mathrm{~cm}$ into the thoracic aorta while the internal carotid artery was blocked. The balloon was inflated with normal saline at $0.5 \mathrm{~atm}$ to $0.7 \mathrm{~atm}$ to distend the artery. Then it was pulled back to the entry point. The entire procedure was repeated three times to denude the endothelium and cause vascular injury. After removing the catheter, the external carotid artery was ligated and the blood circulation of the internal carotid artery restored. In the sham group, only the external carotid artery was ligated. The above surgical operation was done in sterile condition.

2.3. Medications and Grouping. The Yiqihuoxuejiedu formula (Cat. Number 120603), composed of astragalus, salvia, honeysuckle, and other components, was produced by the Chinese Herbal Company of Beijing University of Chinese Medicine (Beijing, China) and the final concentration of crude drug was $1.2 \mathrm{~g} / \mathrm{mL}$.

Rats with balloon injury were randomly divided into three groups: the model group, the Atorvastatin group, and the Yiqihuoxuejiedu group. The sham group served as a control. Rats in the Atorvastatin group were orally administered with $13.33 \mathrm{mg} / \mathrm{kg} / \mathrm{d}$ of Atorvastatin calcium (Pfizer), while they were administered with $12 \mathrm{~g} / \mathrm{kg}$ in the Yiqihuoxuejiedu group and $10 \mathrm{~mL} / \mathrm{kg} / \mathrm{d}$ distilled water in the sham and model groups. Both doses for the two groups were based on the typical daily clinical dosages for adults, corresponding with 10 times of clinical dosages. Rats were administered once a day and seven days later, perfused with $4 \%$ paraformaldehyde through the left ventricle to fix specimen and make paraffin sections.

2.4. Histomorphometric Analysis. Carotid artery sections $(5 \mu \mathrm{m})$ were stained with hematoxylin-eosin. Next, the sections were examined with a microscope (magnification $\times 100$ ) and photographed for morphological analysis. Image analysis software (Image-Pro Plus 6.0) was used to analyze the following morphological indicators: lumen radius (lumen perimeter $/ 2 \pi$ ), neointimal thickness (internal elastic membrane perimeter $/ 2 \pi$ - lumen perimeter $/ 2 \pi$ ), and adventitial area (total area of vessel - area circled by external elastic membrane).

2.5. Radioimmunoassay Measurement and Analysis of CRP. For radioimmunoassay of CRP, a nonequilibrium method was used. The corresponding antibody was used to the standard and sample solutions, mixed well, and placed at $4^{\circ} \mathrm{C}$ 


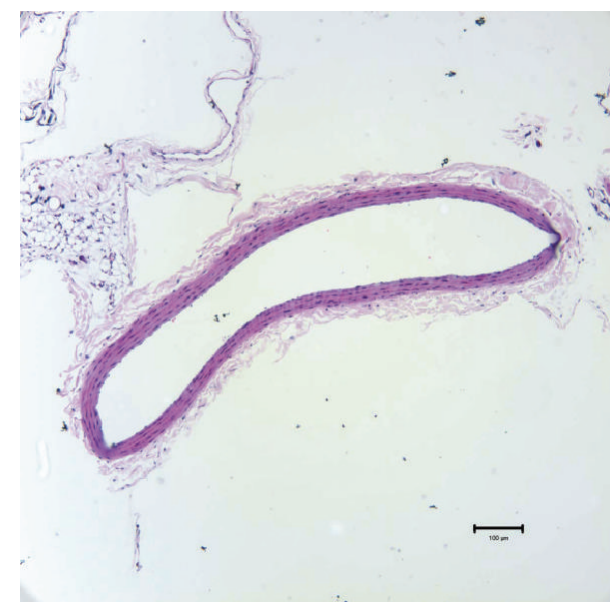

(a)

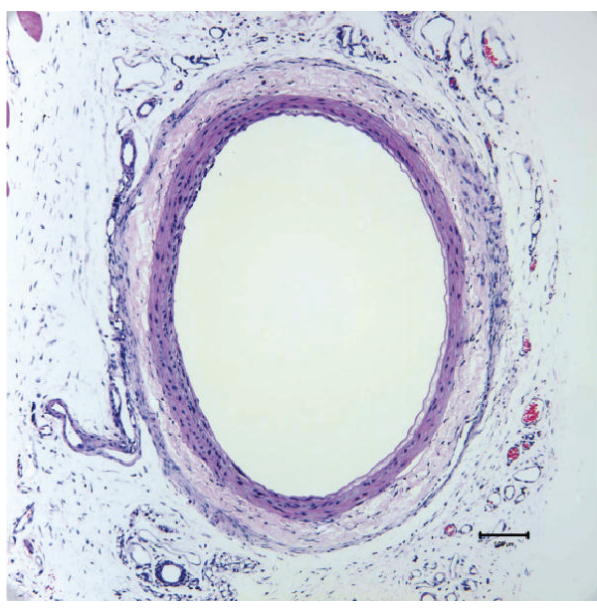

(c)

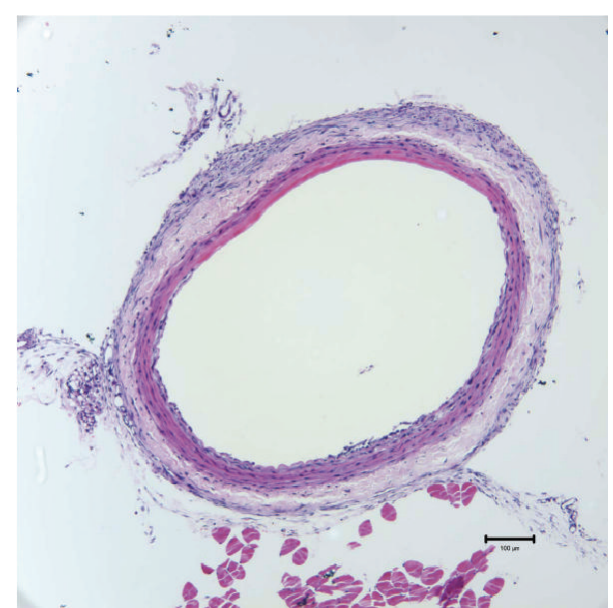

(b)

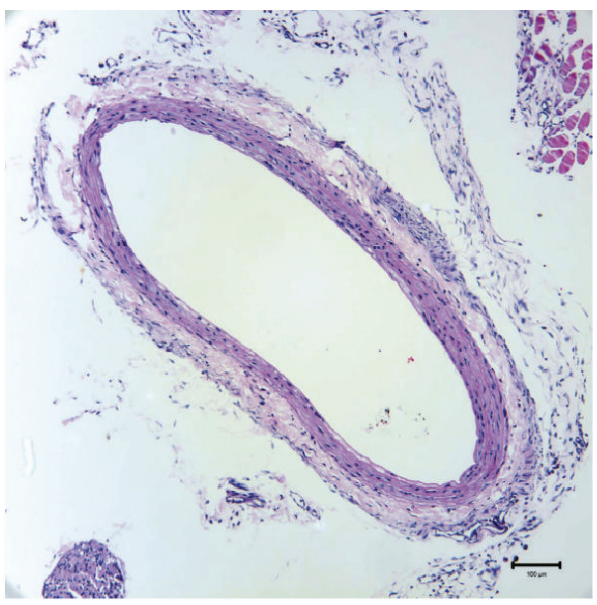

(d)

FIGURE 1: Left common carotid artery slices with HE 7 days after injury. (a) Sham group, (b) model group, (c) Atorvastatin group, and (d) Yiqihuoxuejiedu group.

for $24 \mathrm{~h}$. Next, 125I-VIP (125I-SP, 125I-SS, Beijing Huaying Biotechnology Institute) was added, mixed well, and placed at $4^{\circ} \mathrm{C}$ for another $24 \mathrm{~h}$. After that, appropriate secondary antibody was added, mixed thoroughly, and placed at room temperature for $20 \mathrm{~min}$. Then samples were centrifuged $3500 \mathrm{r}$ for $20 \mathrm{~min}$, and the supernatants were discarded. The radionuclide blink count of precipitation was tested and converted to $\mathrm{mg} / \mathrm{L}$ according to the standard curve.

2.6. Immunohistochemistry Measurement and Analysis of $M C P-1, C D 68$, and Cx43. Different antibodies were used to detect the expressions of MCP-1, CD68, and Cx43 in the vascular wall. All samples were repaired in a microwave and incubated in $0.3 \%$ hydrogen peroxide before using primary antibodies (MCP-1, Abcam, UK; CD68 and Cx43, Santa Cruz Biotechnology, USA) and secondary antibody-HRP multimer (Zhongshan Golden Bridge Biotechnology Company, China). Samples were then visualized with diaminobenzidine substrate. The positive expressions of $\mathrm{CD} 68, \mathrm{Cx} 43$, and MCP-1 appeared as brownish-yellow or a brown granulation in the cytoplasm. The typical images were captured with
SPOT V3.0II software, and the average optical density or the positive area was measured and analyzed by image-Pro Plus 6.0 .

2.7. Statistical Analysis. Mean differences among groups were statistically analyzed using one-way analysis of variance (ANOVA) and between two groups by a TSD test. The level of statistical significance was considered to be $P<0.05$.

\section{Results}

3.1. Lumen Radius and Changes of Neointimal Thickness. Seven days after balloon injury, there was small reduction of lumen radius in the model group but had no significant difference compared with the sham group, neither in Atorvastatin nor in Yiqihuoxuejiedu groups. Intimal hyperplasia appeared obviously in the model group compared to the sham group $(P<0.01)$. Compared with the model group, the Yiqihuoxuejiedu formula could reduce neointimal thickness $(P<0.01$, Figures 1 and 2$)$. 


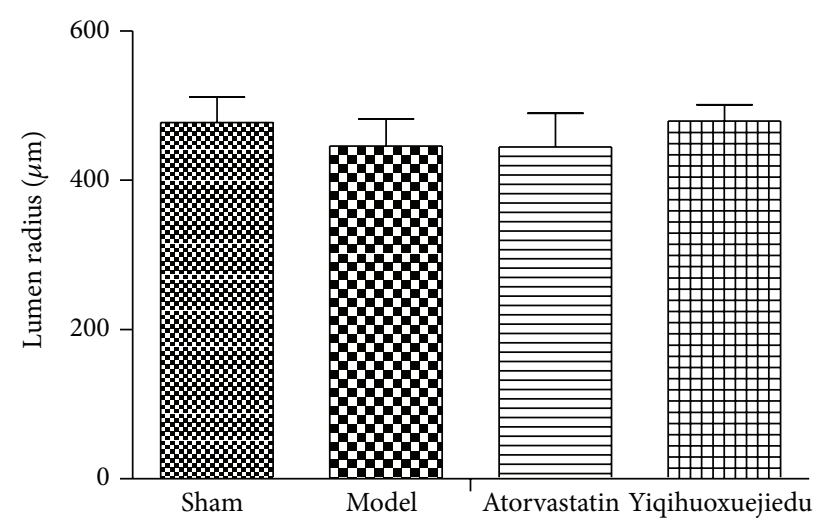

(a)

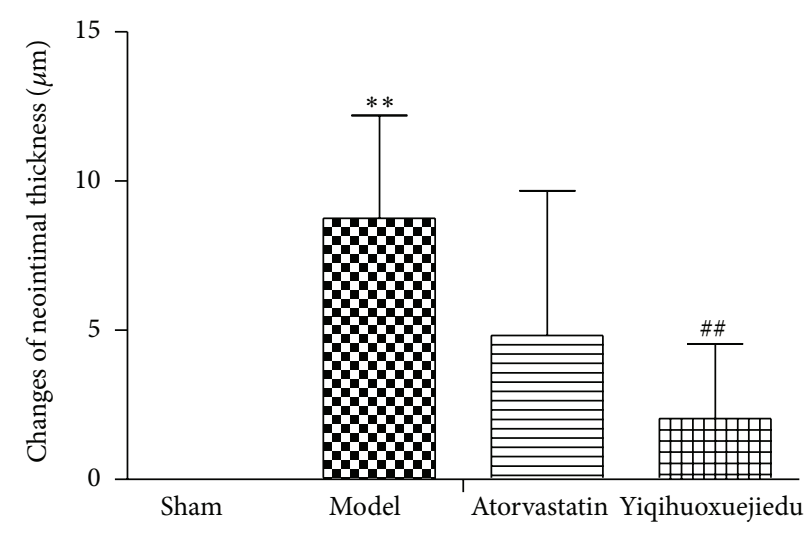

(b)

FIGURE 2: Lumen radius and changes of neointimal thickness 7 days after balloon injury. (a) Lumen radius. (b) Changes of neointimal thickness. ${ }^{* *}$ Compared with sham group, $P<0.01 .{ }^{\# \#}$ Compared with model group, $P<0.01$.

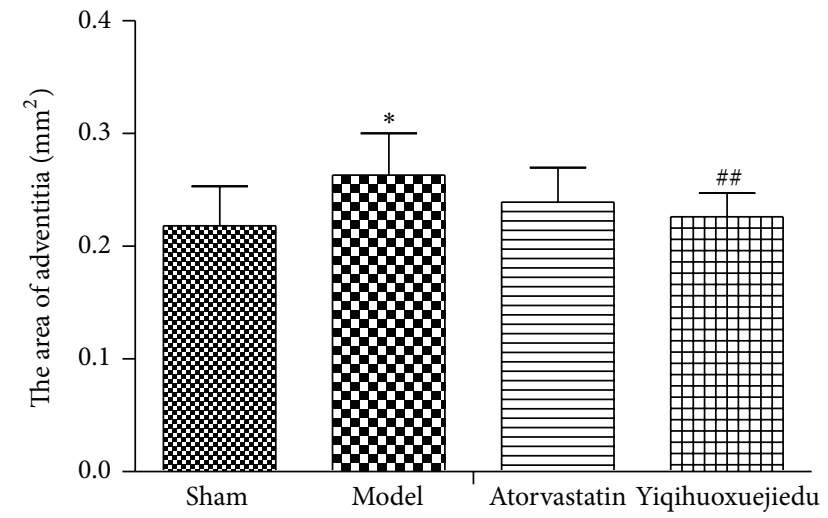

Figure 3: The area of adventitia 7 days after balloon injury. ${ }^{*}$ Compared with sham group, $P<0.05$. ${ }^{\#}$ Compared with model group, $P<0.01$.

3.2. The Area of the Adventitia. There was a significant increase in the area of the adventitia in model group $(P<$ 0.05). Compared with the model group, the area of the adventitia in the Yiqihuoxuejiedu group was decreased $(P<$ 0.01 , Figures 1 and 3 ).

3.3. The Concentration of CRP in Serum. At the early period of injury, CRP increased markedly in the serum, especially in the model group $(P<0.01)$. The Yiqihuoxuejiedu formula decreased CRP $(P<0.01)$, while Atorvastatin only had a trend in reducing CRP (Figure 4).

3.4. The Expression of MCP-1 in Vascular Wall. Immunohistochemistry showed that the expression of MCP-1 in the three layers of vascular wall increased after balloon injury, especially in the adventitia of the model group. The average optical density (OD) in the treatment groups was all decreased; the adventitial positive expression in the Atorvastatin group had an apparent reduction compared with the model group $(P<$ 0.01 , Figure 5).

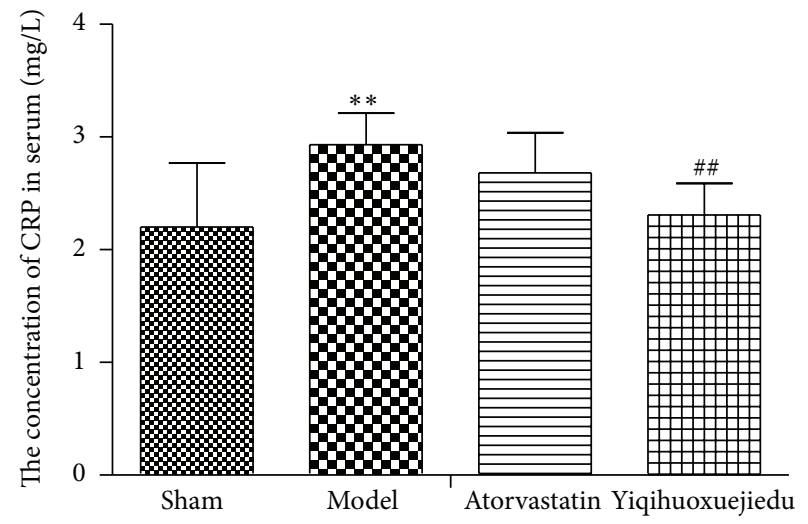

FIgURE 4: The concentration of CRP in serum. ${ }^{* *}$ Compared with sham group, $P<0.01$. ${ }^{\# \#}$ Compared with model group, $P<0.01$.

3.5. The Expression of CD68 in Vascular Wall. Compared with the sham group, CD68 expression of media and adventitia in the model group had a significant increase $(P<0.05$ for media, $P<0.01$ for adventitia). The Yiqihuoxuejiedu formula inhibited positive expression of CD68 in the adventitia $(P<$ $0.01)$, and it had a stronger effect than Atorvastatin $(P<0.05$, Figure 6).

3.6. The Expression of Cx43 in Vascular Wall. There were no significant changes in the average $\mathrm{OD}$ in the vascular wall when determining Cx43 expression. However, the positive areas of $\mathrm{Cx} 43$ in surgery groups were increased, and there was significant difference between the model group and the sham group $(P<0.05)$. Compared with model group, the positive area of $\mathrm{Cx} 43$ in adventitia significantly decreased in the Yiqihuoxuejiedu group $(P<0.05)$. We observed the same trend in the media but without any significant differences (Figure 7). 


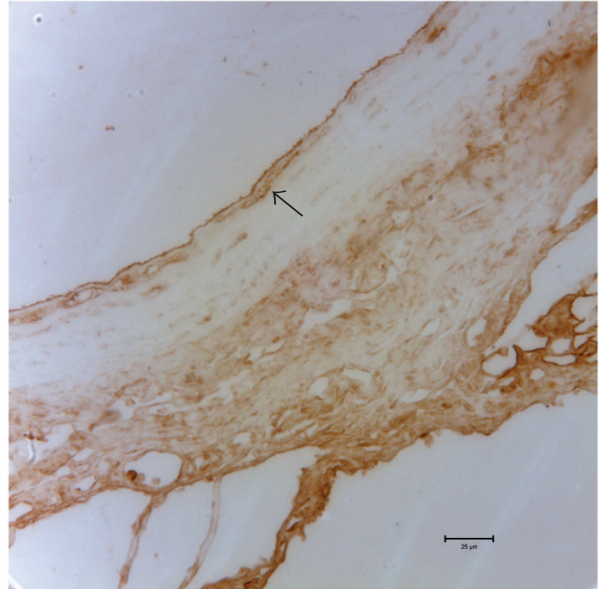

(a)

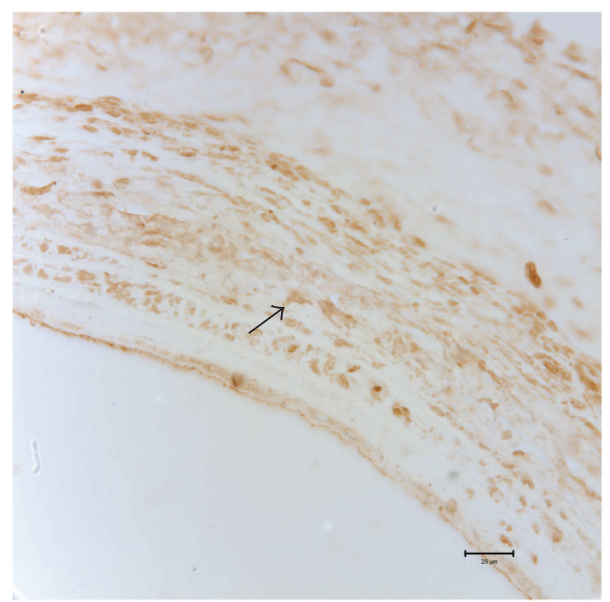

(c)

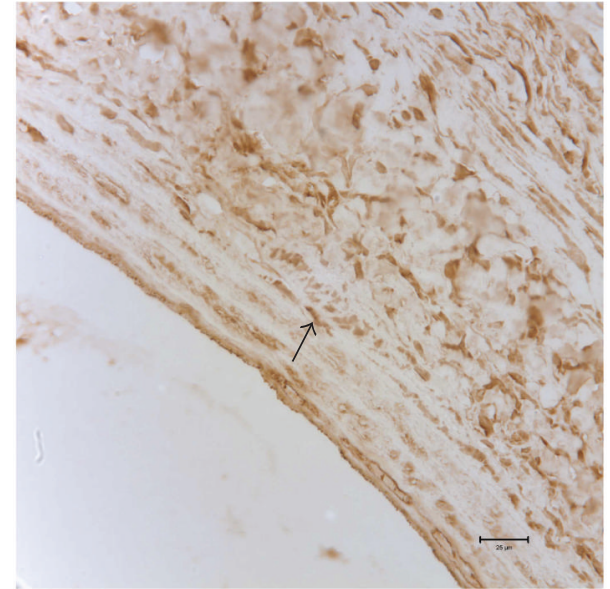

(b)

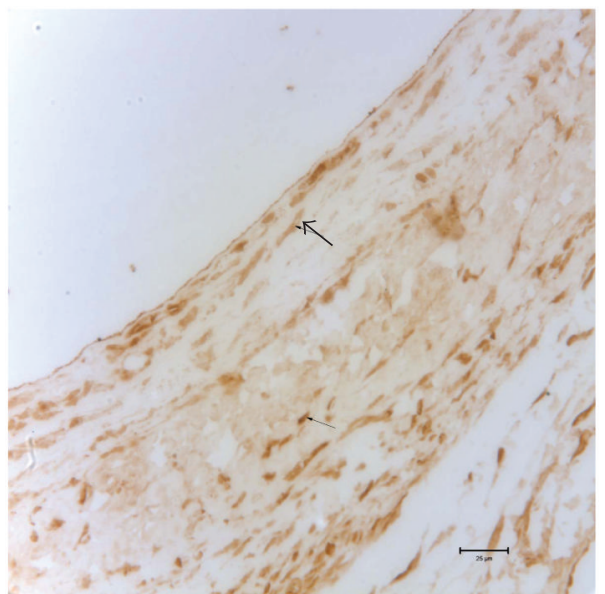

(d)

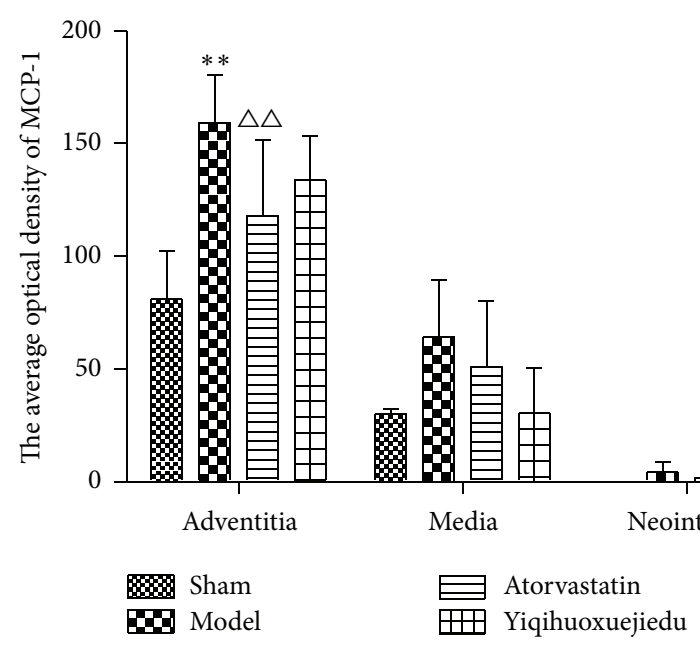

FIGURE 5: The expression of MCP-1 in vascular wall. (a) Sham group, (b) model group, (c) Atorvastatin group, and (d) Yiqihuoxuejiedu group. ${ }^{* *}$ Compared with sham group, $P<0.01$. ${ }^{\# \#}$ Compared with model group, $P<0.01$.

\section{Discussion}

PCI can lead to two types of vascular remodeling, positive remodeling and negative remodeling [26]. Positive remodeling means that the vessels provide compensatory dilation, and the lumen diameter does not change significantly, while negative remodeling means that vessels shrink, and the lumen diameter narrows. In the early remodeling, adventitial fibroblasts play principal roles, although VSMCs also contribute to the pathological change. In this 


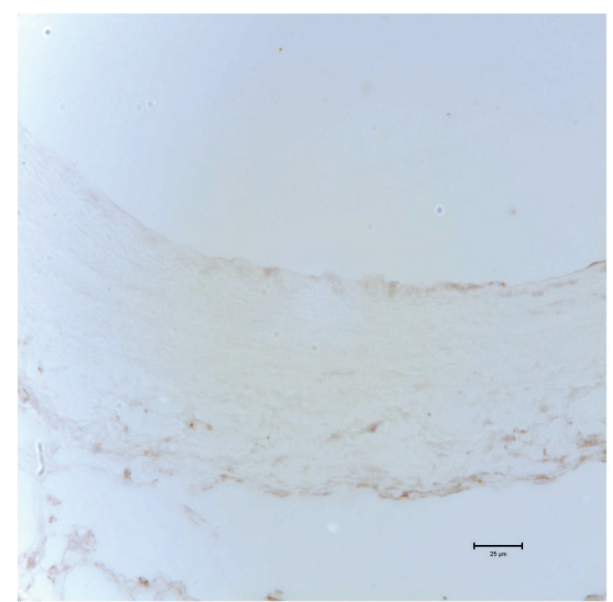

(a)

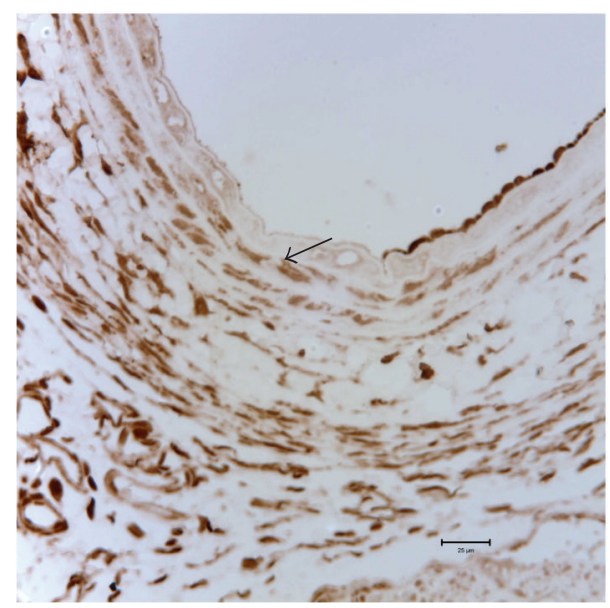

(c)

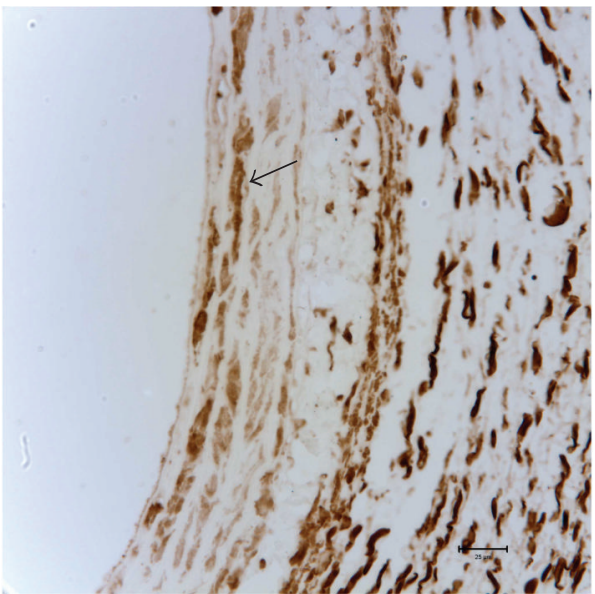

(b)

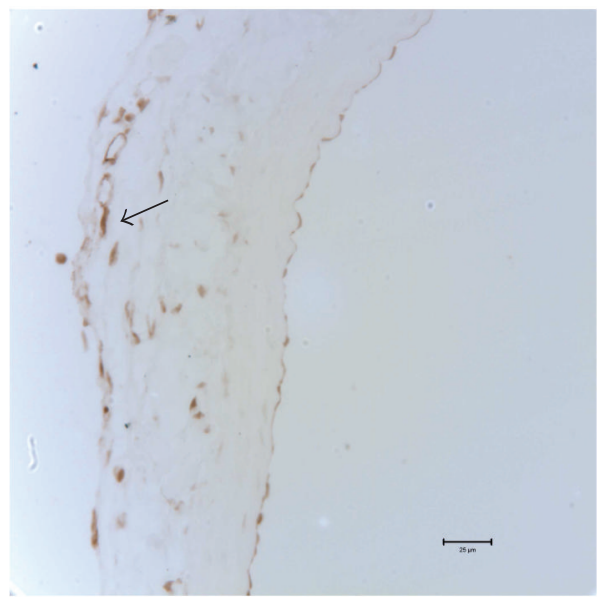

(d)

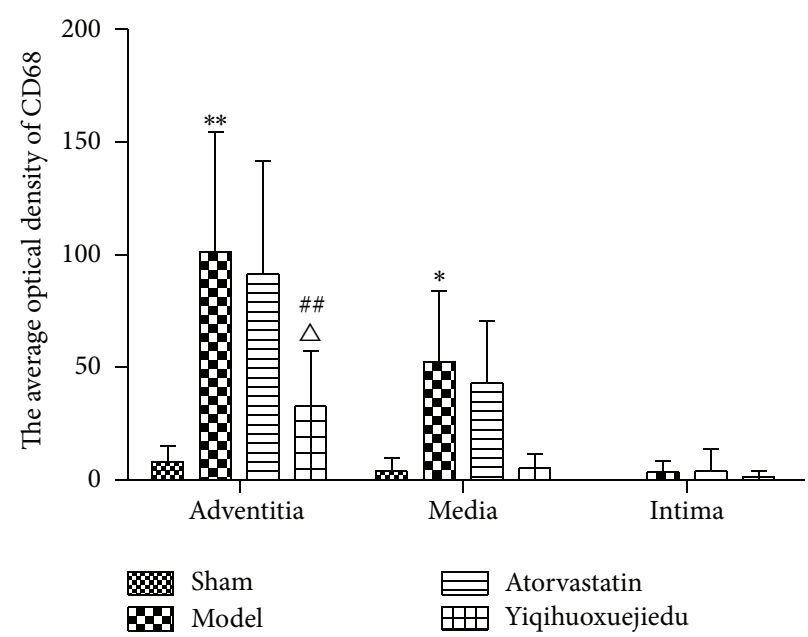

FIGURE 6: The expression of CD68 in vascular wall. (a) Sham group, (b) model group, (c) Atorvastatin group, and (d) Yiqihuoxuejiedu group. ${ }^{* *}$ Compared with sham group, $P<0.01$. ${ }^{\# \#}$ Compared with model group, $P<0.01 .{ }^{\triangle}$ Compared with Atorvastatin group, $P<0.05$.

study, neointimal thickness slowly increased and lumen showed no stenosis, but the adventitial area rapidly grew in model animals. Thus, positive remodeling dominated vascular remodeling at the early phase of intimal injury. The
Yiqihuoxuejiedu formula could inhibit neointimal thickness and reduce the adventitial area. Our previous study demonstrated that $\alpha$-SMA expression of the adventitia increased in model group at 7 days after balloon injury, which indicated 


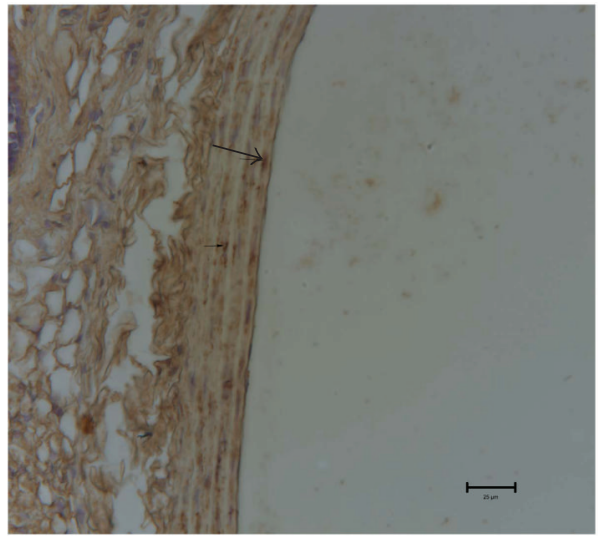

(a)

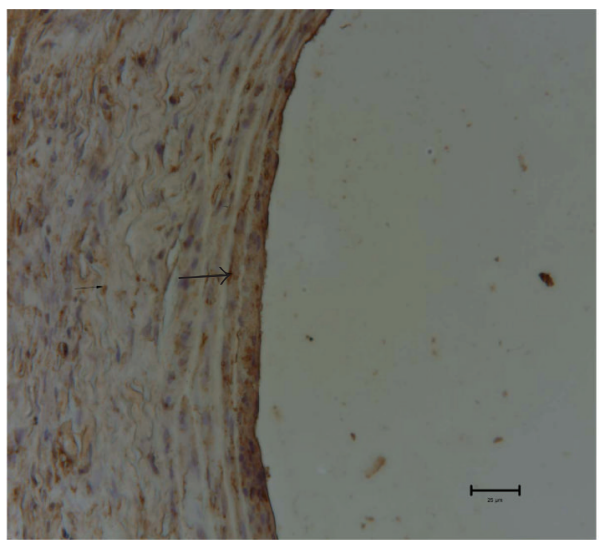

(c)

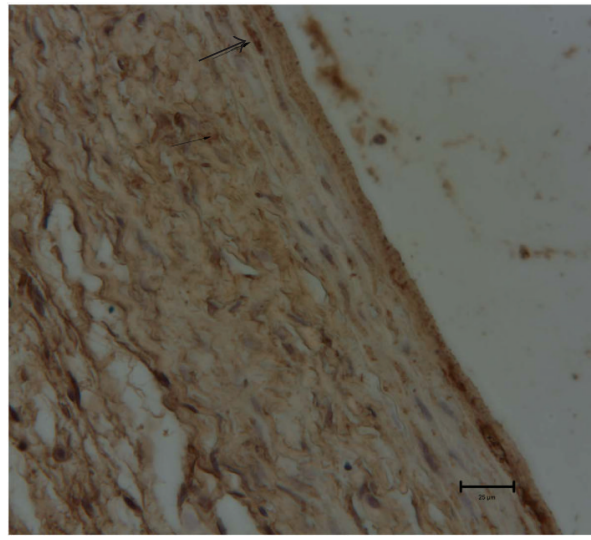

(b)

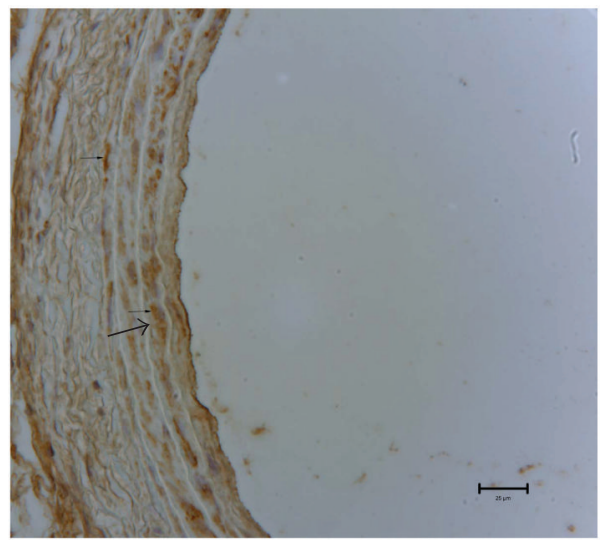

(d)

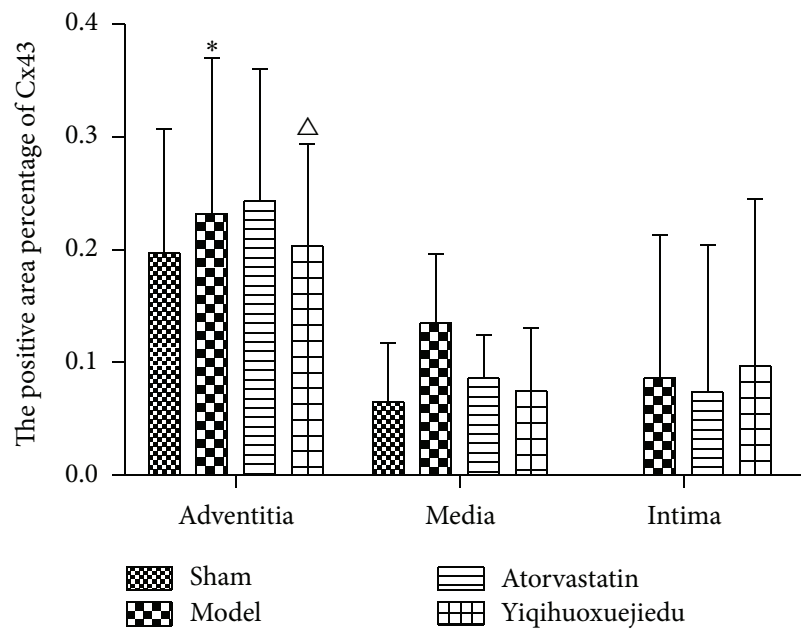

Figure 7: The expression of Cx43 in vascular wall. (a) Sham group, (b) model group, (c) Atorvastatin group, and (d) Yiqihuoxuejiedu group. ${ }^{*}$ Compared with sham group, $P<0.05$. ${ }^{\#}$ Compared with model group, $P<0.05$.

activation and proliferation of the adventitial fibroblasts [25].

C-reactive protein (CRP) not only serves as one of the most widely known biomarkers of cardiovascular disease and underlying inflammation but also promotes activation of endothelial cells and monocytes, leading to vascular remodeling $[27,28]$. The concentration of CRP increases when inflammation occurs in the adventitia [8]. In our study, CRP increased highly in serum, particularly in the model group $(P<0.01)$, while the Yiqihuoxuejiedu formula could diminish CRP $(P<0.01)$.

Lesion of the intima can lead to an early inflammatory response, including infiltration of macrophages and neutrophils and the release of inflammatory factors, especially 
in the adventitia [29]. MCP-1 is secreted by monocytesmacrophages and adventitial fibroblasts [30, 31], and it can stimulate VSMCs to proliferate and migrate [32, 33] and promote monocytes to gather in the adventitia, leading to AFs proliferation and adventitial thickness [7]. In our study, MCP-1 expression increased in all the three layers of the vessel wall and markedly increased in the adventitia $(P<0.01)$. The concentration of MCP-1 was higher in the adventitia than in the media and neointima, indicating severe adventitial inflammation after vascular injury. The positive medicine, Atorvastatin, significantly reduced MCP-1 expression to restore the body's balance between proinflammatory and anti-inflammatory responses [34], but the Yiqihuoxuejiedu formula had no effect on MCP-1.

Thirty minutes after intimal injury, the inflammatory cells can be detected in the adventitia, with earlier time and higher concentration than in the media [6]. Macrophages can release cytokines and promote neointimal hyperplasia [35-37]. CD68 is a special marker of macrophages. Our study showed that macrophages infiltrated into the media and adventitia, particularly in the latter. The Yiqihuoxuejiedu formula limited the infiltration of macrophages dramatically, and its effect was better than that of Atorvastatin $(P<0.05)$, while Atorvastatin failed to do this.

GJs are important channels for exchanging matter, energy, and information between cells. They are closely associated with vascular function. Connexins (Cxs) are the main components of GJs, and Cx43 is expressed mostly in the vascular wall $[16,38]$. Previous studies have indicated that $\mathrm{Cx} 43$ in vessel wall increased after PCI and played a promoting role in vascular remodeling by encouraging VSMCs to proliferate and be activated together, leading to inflammation in injured vessels [39-41]. One of the drug targets is to reduce the level of Cxs and communication among cells to prevent and treat remodeling. The present study showed that $\mathrm{Cx} 43$ expression increased in the vessel wall, especially in the adventitia, and cell communication strengthened, leading to cell proliferation and inflammation. Yiqihuoxuejiedu formula significantly reduced $\mathrm{Cx} 43(P<$ 0.05). Atorvastatin downregulated $\mathrm{Cx} 43$, but the change was not significant. Other studies found different results with Atorvastatin, mostly showing that the drug caused limited Cx43 expression in VSMCs to achieve an antiproliferative role [23]. Different drug doses may account for the different results. We used 10 times the clinical dose in our study, but other studies used higher doses $[42,43]$. Thus, it is unclear whether stains have an effective role in GJs under clinical doses.

Yiqihuoxuejiedu formula is composed of astragalus, salvia, honeysuckle, and other components based on Chinese medicine principle of strengthening qi, activating the blood plus detoxication. Our previous studies have demonstrated that this formula reduced levels of lipids and TGF- $\beta$ [24], limited neointimal hyperplasia, and diminished the collagen deposition in neointimal formation [44]. Astragalus, salvia, and tetramethylpyrazine, the main ingredients, play effective roles in anti-inflammation, downregulating MCP-1 and inhibiting SMC proliferation and migration [45-50]. All of these targets are the vital pharmacological bases for the Yiqihuoxuejiedu formula to inhibit neointimal formation and reduce macrophages infiltration and $\mathrm{Cx} 43$ expression in present study.

\section{Conclusion}

In summary, the injured vessel presents positive remodeling with slight neointimal hyperplasia and remarkable adventitial remodeling, and it also elicits inflammatory reactions with high expressions of MCP-1, CD68, and Cx43, particularly in the adventitia at the early phase of intimal injury. Yiqihuoxuejiedu formula restrained expression of CD68 and Cx43 remarkably in the adventitia and reduced the concentration of CRP and diminished intimal hyperplasia and area of the adventitia. These results indicated that this formula could inhibit vascular remodeling by limiting macrophages infiltration in the adventitia, depressing systemic nonspecific inflammatory response and AFs proliferation, and reducing GJs between cells. This study provides new ideas and methods for the prevention and treatment of vascular remodeling.

\section{Conflict of Interests}

The authors declare that they have no conflict of interests.

\section{Authors' Contribution}

Hong Chang, Huan Lei, Yizhou Zhao, and Ruixue Yang contributed equally to this study.

\section{Acknowledgment}

This paper was supported by the National Natural Science Foundation Project of China (no. 81173234).

\section{References}

[1] J. B. Koenig and I. Z. Jaffe, "Direct role for smooth muscle cell mineralocorticoid receptors in vascular remodeling: novel mechanisms and clinical implications," Current Hypertension Reports, vol. 16, no. 5, article 427, 2014.

[2] B. Yang, R. Janardhanan, P. Vohra et al., "Adventitial transduction of lentivirus-shRNA-VEGF-A in arteriovenous fistula reduces venous stenosis formation," Kidney International, vol. 85, no. 2, pp. 289-306, 2014.

[3] K. R. Stenmark, M. E. Yeager, K. C. El Kasmi et al., "The adventitia: essential regulator of vascular wall structure and function," Annual Review of Physiology, vol. 75, pp. 23-47, 2013.

[4] A. Fedorov, A. Kostareva, J. Raud, J. Roy, U. Hedin, and A. Razuvaev, "Early changes of gene expression profiles in the rat model of arterial injury," Journal of Vascular and Interventional Radiology, vol. 25, no. 5, pp. 789.e7-796.e7, 2014.

[5] W. Lan, F. Yang, Z. Li et al., "Human urine kininogenase attenuates balloon-induced intimal hyperplasia in rabbit carotid artery through transforming growth factor $\beta 1 / \mathrm{Smad} 2 / 3$ signaling pathway," Journal of Vascular Surgery, 2015.

[6] E.-I. Okamoto, T. Couse, H. De Leon et al., "Perivascular inflammation after balloon angioplasty of porcine coronary arteries," Circulation, vol. 104, no. 18, pp. 2228-2235, 2001. 
[7] B. C. Tieu, X. Ju, C. Lee et al., "Aortic adventitial fibroblasts participate in angiotensin-induced vascular wall inflammation and remodeling," Journal of Vascular Research, vol. 48, no. 3, pp. 261-272, 2011.

[8] P. Liu, C. Zhang, Y. X. Zhao et al., "Gax gene transfer inhibits vascular remodeling induced by adventitial inflammation in rabbits," Atherosclerosis, vol. 212, no. 2, pp. 398-405, 2010.

[9] M. Zhang, N. Cresswell, F. Tavora, E. Mont, Z. Zhao, and A. Burke, "In-stent restenosis is associated with neointimal angiogenesis and macrophage infiltrates," Pathology Research and Practice, vol. 210, no. 12, pp. 1026-1030, 2014.

[10] G. Li, S.-J. Chen, S. Oparil, Y.-F. Chen, and J. A. Thompson, "Direct in vivo evidence demonstrating neointimal migration of adventitial fibroblasts after balloon injury of rat carotid arteries," Circulation, vol. 101, no. 12, pp. 1362-1365, 2000.

[11] S. Sartore, A. Chiavegato, E. Faggin et al., "Contribution of adventitial fibroblasts to neointima formation and vascular remodeling: from innocent bystander to active participant," Circulation Research, vol. 89, no. 12, pp. 1111-1121, 2001.

[12] A. C. Brisset, B. E. Isakson, and B. R. Kwak, "Connexins in vascular physiology and pathology," Antioxidants \& Redox Signaling, vol. 11, no. 2, pp. 267-282, 2009.

[13] N. J. Severs, S. Rothery, E. Dupont et al., "Immunocytochemical analysis of connexin expression in the healthy and diseased cardiovascular system," Microscopy Research and Technique, vol. 52, no. 3, pp. 301-322, 2001.

[14] M. J. A. van Kempen and H. J. Jongsma, "Distribution of connexin37, connexin 40 and connexin 43 in the aorta and coronary artery of several mammals," Histochemistry and Cell Biology, vol. 112, no. 6, pp. 479-486, 1999.

[15] T. L. Little, E. C. Beyer, and B. R. Duling, "Connexin 43 and connexin 40 gap junctional proteins are present in arteriolar smooth muscle and endothelium in vivo," American Journal of Physiology-Heart and Circulatory Physiology, vol. 268, no. 2, part 2, pp. H729-H739, 1995.

[16] J.-A. Haefliger, P. Nicod, and P. Meda, "Contribution of connexins to the function of the vascular wall," Cardiovascular Research, vol. 62, no. 2, pp. 345-356, 2004.

[17] L. P. Véliz, F. G. González, B. R. Duling, J. C. Sáez, and M. P. Boric, "Functional role of gap junctions in cytokine-induced leukocyte adhesion to endothelium in vivo," The American Journal of Physiology-Heart and Circulatory Physiology, vol. 295, no. 3, pp. H1056-H1066, 2008.

[18] B. R. Kwak, N. Veillard, G. Pelli et al., "Reduced connexin43 expression inhibits atherosclerotic lesion formation in lowdensity lipoprotein receptor-deficient mice," Circulation, vol. 107, no. 7, pp. 1033-1039, 2003.

[19] T. Matsushita, A. Rama, N. Charolidi, E. Dupont, and N. J. Severs, "Relationship of connexin43 expression to phenotypic modulation in cultured human aortic smooth muscle cells," European Journal of Cell Biology, vol. 86, no. 10, pp. 617-628, 2007.

[20] X. Han, M. Chen, T. Hong et al., "Lentivirus-mediated RNAi knockdown of the gap junction protein, $\mathrm{Cx} 43$, attenuates the development of vascular restenosis following balloon injury," International Journal of Molecular Medicine, vol. 35, no. 4, pp. 885-892, 2015.

[21] G. N. Levine, E. R. Bates, J. C. Blankenship et al., "2011 ACCF/AHA/SCAI Guideline for Percutaneous Coronary Intervention. A report of the American College of Cardiology Foundation/American Heart Association Task Force on Practice
Guidelines and the Society for Cardiovascular Angiography and Interventions," Journal of the American College of Cardiology, vol. 58, no. 24, pp. e44-e122, 2011.

[22] N. Tahara, A. Tahara, J. Narula, and T. Imaizumi, "Statin therapy resolves coronary artery inflammation," JACC: Cardiovascular Imaging, vol. 6, no. 10, pp. 1119-1120, 2013.

[23] J. Shen, L.-H. Wang, L.-R. Zheng, J.-H. Zhu, and S.-J. Hu, "Lovastatin inhibits gap junctional communication in cultured aortic smooth muscle cells," Journal of Cardiovascular Pharmacology and Therapeutics, vol. 15, no. 3, pp. 296-302, 2010.

[24] M. J. Zhao, S. R. Wang, Q. M. Li et al., "Experimental study of Yiqihuoxuejiedu decoction on inhibiting restenosis after percutaneous transluminal angiography in rabbits," Chinese Journal of Experimental Traditional Medical Formulae, vol. 13, no. 3, pp. 32-36, 2007.

[25] M.-J. Zhao, A.-M. Wu, J. Wang et al., "Yiqihuoxuejiedu formula inhibits vascular remodeling by reducing proliferation and secretion of adventitial fibroblast after balloon injury," EvidenceBased Complementary and Alternative Medicine, vol. 2014, Article ID 849167, 8 pages, 2014.

[26] G. H. Gibbons and V. J. Dzau, "The emerging concept of vascular remodeling," The New England Journal of Medicine, vol. 330, no. 20, pp. 1431-1438, 1994.

[27] F. G. Hage, "C-reactive protein and Hypertension," Journal of Human Hypertension, vol. 28, no. 7, pp. 410-415, 2014.

[28] N. Mahajan and V. Dhawan, "In vitro modulation of peroxisome proliferator-activated receptor- $\gamma$ and its genes by C-reactive protein. Role of atorvastatin," Archives of Medical Research, vol. 41, no. 3, pp. 154-161, 2010.

[29] M.-L. Kortelainen and K. Porvari, "Adventitial macrophage and lymphocyte accumulation accompanying early stages of human coronary atherogenesis," Cardiovascular Pathology, vol. 23, no. 4, pp. 193-197, 2014.

[30] K. G. Battiston, B. Ouyang, R. S. Labow, C. A. Simmons, and J. P. Santerre, "Monocyte/macrophage cytokine activity regulates vascular smooth muscle cell function within a degradable polyurethane scaffold," Acta Biomaterialia, vol. 10, no. 3, pp. 1146-1155, 2014.

[31] B. C. Tieu, C. Lee, H. Sun et al., "An adventitial IL-6/MCP1 amplification loop accelerates macrophage-mediated vascular inflammation leading to aortic dissection in mice," The Journal of Clinical Investigation, vol. 119, no. 12, pp. 3637-3651, 2009.

[32] Y. J. Yang, L. S. Wu, B. Shu, and M. Z. Qian, "MCPIP1 mediates MCP-1-induced vascular smooth muscle cell proliferation," Acta Physiologica Sinica, vol. 65, no. 6, pp. 616-622, 2013.

[33] V. Kundumani-Sridharan, N. K. Singh, S. Kumar, R. Gadepalli, and G. N. Rao, "Nuclear factor of activated T cells c1 mediates p21-activated kinase 1 activation in the modulation of chemokine-induced human aortic smooth muscle cell F-actin stress fiber formation, migration, and proliferation and injuryinduced vascular wall remodeling," The Journal of Biological Chemistry, vol. 288, no. 30, pp. 22150-22162, 2013.

[34] B. Mirjanic-Azaric, M. Rizzo, L. Sormaz et al., "Atorvastatin in stable angina patients lowers CCL2 and ICAM1 expression: pleiotropic evidence from plasma mRNA analyses," Clinical Biochemistry, vol. 46, no. 15, pp. 1526-1531, 2013.

[35] J. R. Hoch, V. K. Stark, N. van Rooijen, J. L. Kim, M. P. Nutt, and T. F. Warner, "Macrophage depletion alters vein graft intimal hyperplasia," Surgery, vol. 126, no. 2, pp. 428-437, 1999.

[36] J. Yu, C. Fernández-Hernando, Y. Suarez et al., "Reticulon 4B (Nogo-B) is necessary for macrophage infiltration and tissue 
repair," Proceedings of the National Academy of Sciences of the United States of America, vol. 106, no. 41, pp. 17511-17516, 2009.

[37] Y. Kondo, C. C. Jadlowiec, A. Muto et al., "The Nogo-B-PirB axis controls macrophage-mediated vascular remodeling," PLoS ONE, vol. 8, no. 11, Article ID e81019, 2013.

[38] S. Morel and B. R. Kwak, "Vascular connexins in restenosis after balloon injury," Methods in Molecular Biology, vol. 1037, pp. 381398, 2013.

[39] H. Zhong, Q.-H. Hu, E.-B. Wang et al., "Roles of gap junctions in proliferation mediated by Hcy in the spontaneous hypertensive rat vascular smooth muscle cells," Zhongguo ying yong sheng $l i$ xue za zhi, vol. 28, no. 4, pp. 289-293, 2012.

[40] C. N. Joshi, D. N. Martin, P. Shaver, C. Madamanchi, B. J. Muller-Borer, and D. A. Tulis, "Control of vascular smooth muscle cell growth by connexin 43," Frontiers in Physiology, vol. 3, article 220, 2012.

[41] L. Wang, J. Chen, Y. Sun et al., "Regulation of connexin expression after balloon injury: possible mechanisms for antiproliferative effect of statins," American Journal of Hypertension, vol. 18, no. 9, pp. 1146-1153, 2005.

[42] D. D. Li, W. Z. Zhang, Y. Q. Chen, and L. Ma, "Effects of atorvastatin on angiotensin II-inducing ventricular connexin 43 remodeling in rats," South China Journal of Cardiovascular Diseases, vol. 17, no. 1, pp. 55-59, 2011.

[43] L. Li, X. Y. Zhao, G. Q. Liu, P. L. Yang, Y. L. Chen, and W. J. Dong, "The effects of atorvastatin on left ventricular remodeling in spontaneously hypertensive rats," Chinese Journal of Hypertension, vol. 17, no. 7, pp. 636-640, 2009.

[44] M. J. Zhao, S. R. Wang, and Q. M. Li, "Comparative study of Yiqihuoxuejiedu decoction and Xuefuzhuyu capsule on inhibiting extracellular matrix in restenosis," Chinese Journal of Integrative Medicine on Cardio-/Cerebrovascular Disease, vol. 3, no. 12, pp. 1061-1063, 2005.

[45] S. Ohkawara, Y. Okuma, T. Uehara, T. Yamagishi, and Y. Nomura, "Astrapterocarpan isolated from Astragalus membranaceus inhibits proliferation of vascular smooth muscle cells," European Journal of Pharmacology, vol. 525, no. 1-3, pp. 41-47, 2005.

[46] G.-F. Wang, C.-G. Shi, M.-Z. Sun et al., “Tetramethylpyrazine attenuates atherosclerosis development and protects endothelial cells from ox-LDL," Cardiovascular Drugs and Therapy, vol. 27, no. 3, pp. 199-210, 2013.

[47] J. Lu, X. Chen, Y. Zhang et al., "Astragalus polysaccharide induces anti-inflammatory effects dependent on AMPK activity in palmitate-treated RAW264.7 cells," International Journal of Molecular Medicine, vol. 31, no. 6, pp. 1463-1470, 2013.

[48] L. Sun, R. Zhao, L. Zhang et al., "Salvianolic acid A inhibits PDGF-BB induced vascular smooth muscle cell migration and proliferation while does not constrain endothelial cell proliferation and nitric oxide biosynthesis," Molecules, vol. 17, no. 3, pp. 3333-3347, 2012.

[49] S. Xu, P. J. Little, T. Lan et al., "Tanshinone II-A attenuates and stabilizes atherosclerotic plaques in apolipoprotein-E knockout mice fed a high cholesterol diet," Archives of Biochemistry and Biophysics, vol. 515, no. 1-2, pp. 72-79, 2011.

[50] G. Ma, S. Ding, Y. Feng, C. Shen, L. Chen, and Z. Chen, "Tetramethylpyrazine-eluting stents prevented in-stent restenosis in a porcine model," Journal of Cardiovascular Pharmacology, vol. 50, no. 2, pp. 201-205, 2007. 


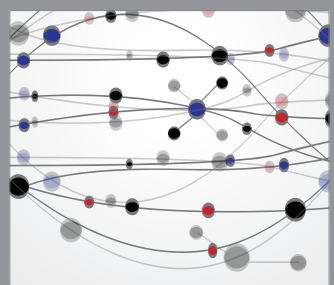

The Scientific World Journal
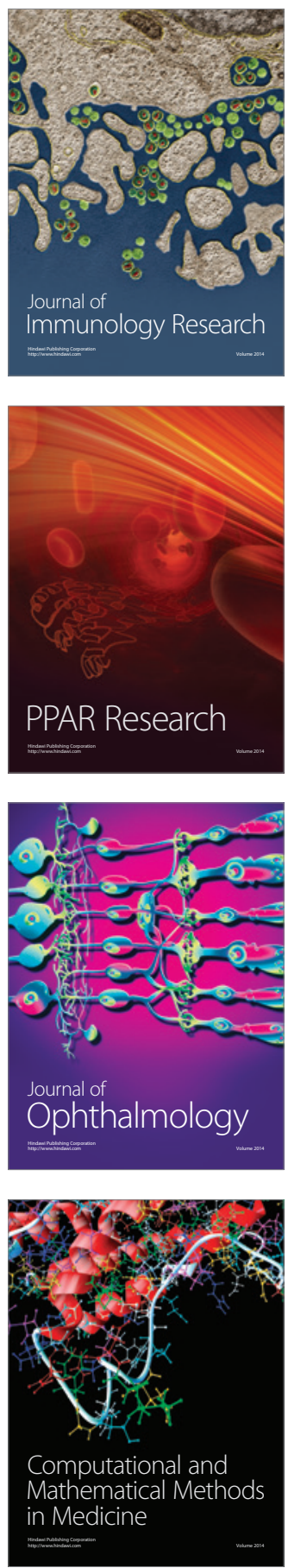

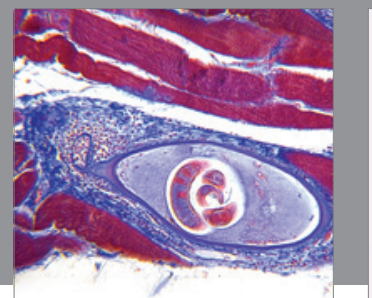

Gastroenterology

Research and Practice
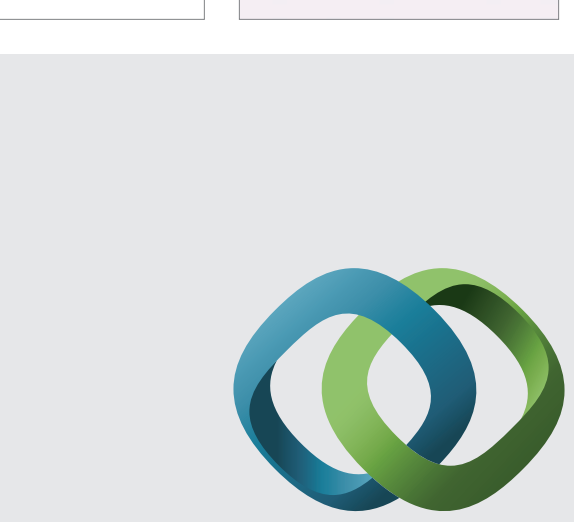

\section{Hindawi}

Submit your manuscripts at

http://www.hindawi.com
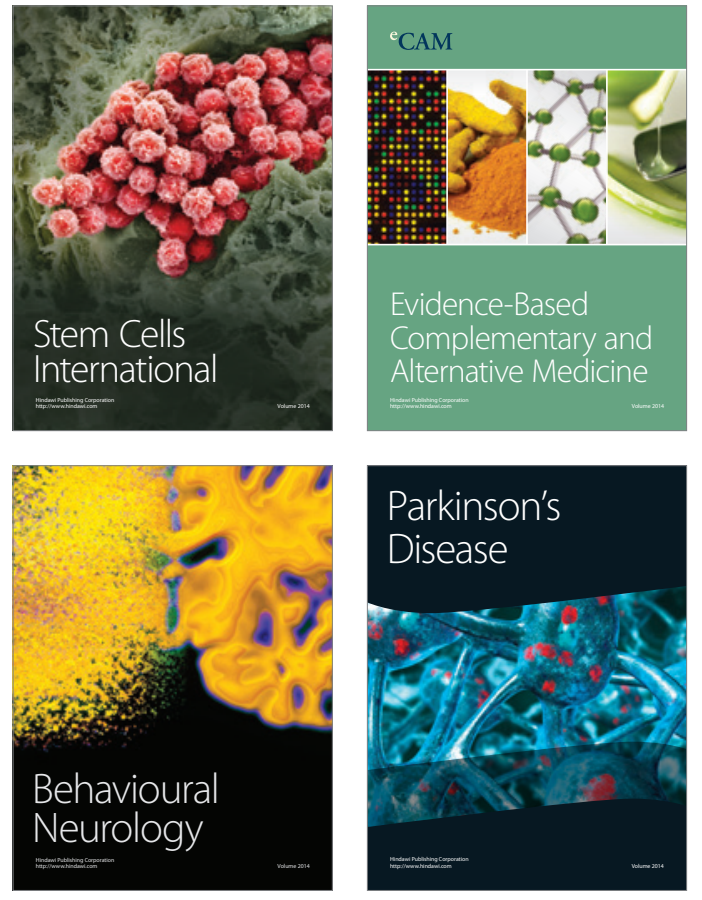
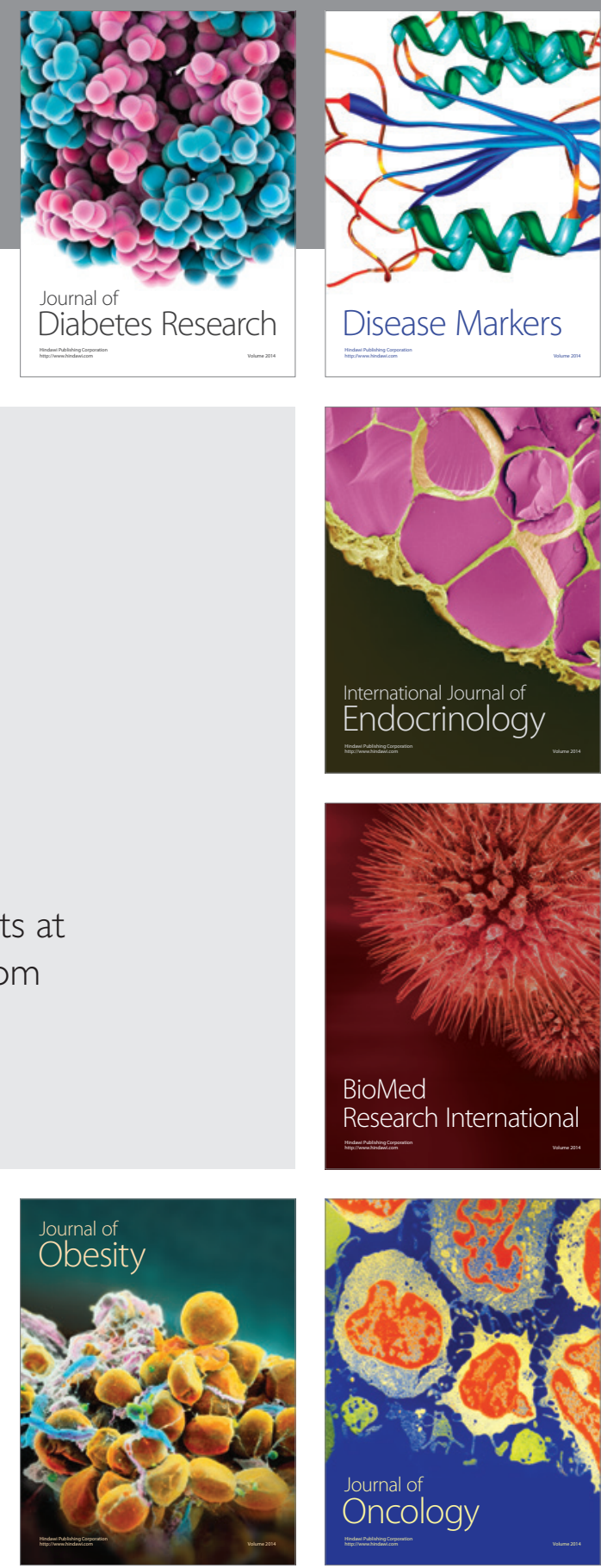

Disease Markers
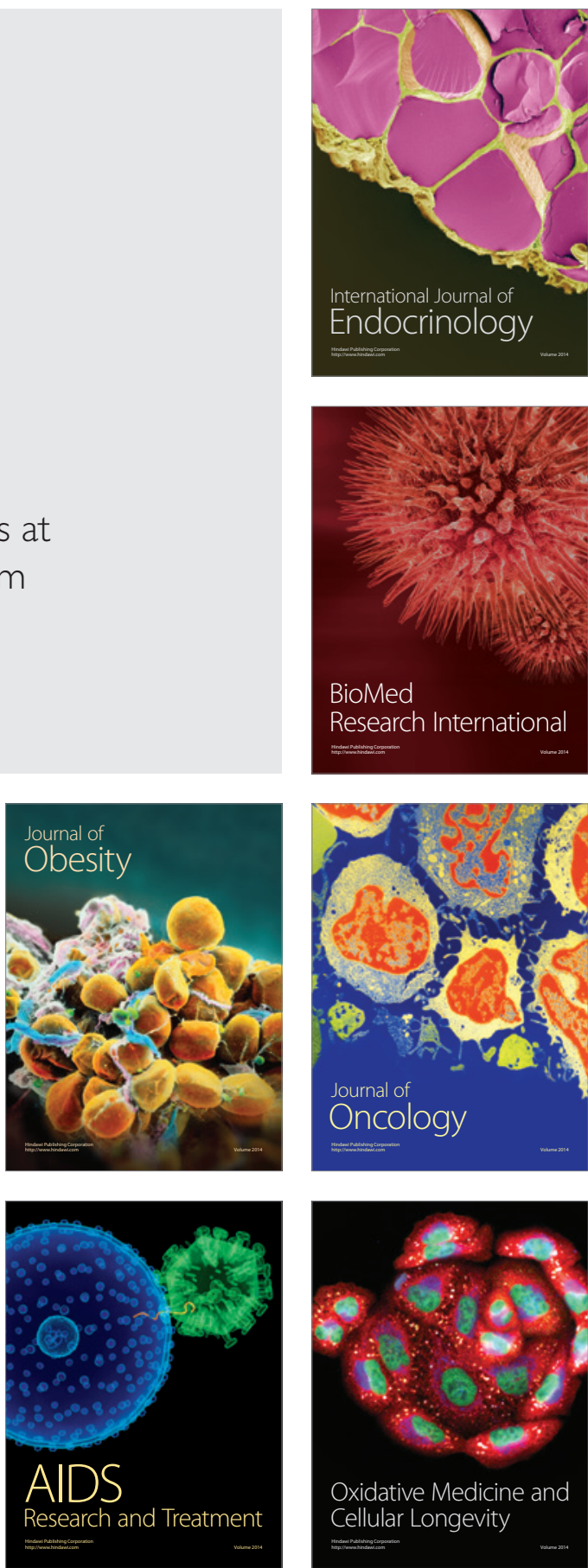